\title{
Apoptosis en las enfermedades cardiovasculares
}

\author{
Javier Díez \\ Departamento de Cardiología y Cirugía Cardiovascular. Clínica Universitaria de Navarra \\ y Unidad de Fisiopatología Vascular. Facultad de Medicina. Universidad de Navarra. Pamplona.
}

\begin{abstract}
La apoptosis constituye una forma de muerte celular con características morfológicas y dinámicas distintas de la muerte por oncosis o por necrosis. Desde el punto de vista de la arquitectura celular, la apoptosis equilibra el efecto de la proliferación celular. Por ello, las alteraciones de la regulación de la apoptosis pueden participar en el desarrollo de numerosas enfermedades. Es el caso de la apoptosis exagerada, que conduce a la atrofia y al fallo de la función de un órgano, o de la apoptosis insuficiente, que propicia el remodelado estructural organotisular. En los últimos años se asiste a una gran expansión en la investigación sobre apoptosis, una investigación en la que también participa la comunidad cardiovascular. Desde muy diversos ámbitos de la medicina cardiovascular se han aportado observaciones sobre el posible papel de la apoptosis en síndromes que oscilan desde los defectos de conducción hasta la insuficiencia cardíaca congestiva, desde la aterosclerosis hasta los aneurismas. No hay duda de que esas observaciones puntuales acabarán configurando conceptos etiopatogénicos y fisiopatológicos que serán la base del diseño de nuevas estrategias diagnósticas y terapéuticas.
\end{abstract}

Palabras clave: Apoptosis. Aterosclerosis. Hipertensión arterial. Insuficiencia cardíaca.

(Rev Esp Cardiol 2000; 53: 267-274)

\section{Apoptosis in Cardiovascular Diseases}

Apoptosis consists of a distinct form of cell death that displays characteristic alterations in cell morphology and cell fate which are different than death due to oncosis or necrosis. In terms of tissue kinetics, apoptosis may be considered a mechanism that counterbalances the effect of cell proliferation by mitotic division. In fact, deregulated apoptosis has been implicated in the development a wide variety of human diseases. Excessive apoptotic cell death may cause organ atrophy and organ failure. On the other hand, insufficient elimination of redundant cells may lead to organ and tissue structural remodeling. In recent years, apoptosis has become a highly fashionable and competitive area of research. Fortunately, it has not escaped the attention of the cardiovascular community. Sightings of apoptosis have been reported from every corner of cardiovascular medicine ranging from conduction system defects to congestive heart failure, and from atherosclerosis to aneurysms. There is no question that these sightings will eventually be converted into mechanistic etiopathogenic and physiopathological insights and will form the basis for designing new diagnostic modalities and novel therapies.

Key words: Apoptosis. Atherosclerosis. Hypertension. Heart failure.

(Rev Esp Cardiol 2000; 53: 267-274)

\section{INTRODUCCIÓN}

Desde las primeras descripciones clásicas de Virchow se reconocía un tipo de muerte celular: la necrosis. Sin embargo, a finales del siglo pasado, Walter Flemming describió un tipo de muerte celular con características morfológicas diferentes, al que denominó cromatólisis. Tuvo que transcurrir casi un siglo hasta

Correspondencia: Dr. J. Díez.

Unidad de Fisiopatología Vascular. Facultad de Medicina.

Universidad de Navarra.

Irunlarrea, s/n. 31080 Pamplona.

Correo electrónico: jadimar@unav.es que este tipo de muerte celular fuese adecuadamente caracterizado por Kerr, quien en 1972 propuso el término apoptosis para denominarlo ${ }^{1}$.

En los últimos 20 años, la apoptosis y la necrosis se han considerado como los dos tipos fundamentales de muerte celular. Sin embargo, Majno y Joris han puesto de manifiesto la inconsistencia de este concepto ${ }^{2}$. En efecto, en términos estrictos, la necrosis representa el conjunto de cambios degradativos en los que culmina cualquier tipo de muerte celular. Así, a la necrosis se llega tanto por un proceso de apoptosis, morfológicamente caracterizado por el arrugamiento y la fragmentación de la célula, como por un proceso de oncosis, en el que la célula se hincha y estalla. Por lo tanto, hoy día cabe distinguir dos tipos fundamentales de muerte celular: el apoptótico y el oncocítico. 


\section{TABLA 1. Principales características diferenciales de la apoptosis y la oncosis que permiten su identificación in situ}

\begin{tabular}{|c|c|c|}
\hline Característica & Apoptosis & Oncosis \\
\hline $\begin{array}{l}\text { Tinción positiva de fragmentos de ADN } \\
\text { de doble hélice usando el TUNEL } \\
\text { u otras técnicas }\end{array}$ & Presente & Ausente \\
\hline $\begin{array}{l}\text { Patrón de distribución del ADN en un gel } \\
\text { de electroforesis }\end{array}$ & Escalonado & Abigarrado \\
\hline $\begin{array}{l}\text { Redistribución de la fosfatidil serina hacia } \\
\text { la superficie externa de la membrana } \\
\text { celular }\end{array}$ & Presente & Ausente \\
\hline $\begin{array}{l}\text { Captación intracelular de trazadores de } \\
\text { permeabilidad de la membrana celular }\end{array}$ & Ausente & Presente \\
\hline
\end{tabular}

Además de presentar una apariencia morfológica distinta, ambos tipos de muerte celular tienen características diferenciales que permiten su identificación (tabla 1). De todas ellas, la más importante es la que se refiere a las alteraciones del ADN. En todo proceso de muerte celular se produce la activación de unas enzimas, las nucleasas, que fragmentan al $\mathrm{ADN}^{3}$. Mientras que en la apoptosis se activan selectivamente ciertas endonucleasas que fragmentan al ADN sólo en sitios internucleosomales, en la oncosis se activan más generalizadamente endonucleasas y exonucleasas, que fragmentan al ADN tanto en sitios internucleosomales como nucleosomales.

Otra diferencia fundamental entre ambos tipos de muerte celular viene dado por su coste energético. Así, mientras que en el proceso de apoptosis se requiere energía para la síntesis de ARN y de proteínas, la on- cosis no consume energía. Por ello, actualmente se acepta que la disponibilidad de ATP de una célula puede determinar que, ante un mismo estímulo, ésta muera vía apoptosis o vía oncosis ${ }^{4}$.

\section{ASPECTOS MOLECULARES DEL PROCESO DE LA APOPTOSIS}

En el proceso de la apoptosis hay que distinguir cuatro fases distintas $^{5-7}$ (fig. 1): la puesta en marcha, la activación de los mediadores intracelulares, la acción de los ejecutores y los cambios finales que conducen a su resolución.

Dos son los tipos de estímulos que pueden poner en marcha la apoptosis: estímulos que actúan desde el exterior de la célula y estímulos originados en la propia célula. Entre los primeros hay que considerar varios: a) la carencia de nutrientes y de factores de crecimiento; $b$ ) factores como el TNF que actúan a través de receptores de la membrana celular acoplados al sistema del Fas, que a su vez activa a una serie de proteínas del llamado «dominio de muerte» (TRADD; FADD, FLICE); c) hormonas y factores vasoactivos, como la angiotensina II, que actúan a través de receptores específicos de la membrana celular que activan el programa genético de muerte de la propia célula regulado por las proteínas de la familia $\mathrm{Bcl}-2, \mathrm{y} d$ ) factores que inciden directamente sobre la cromatina nuclear, como en el caso de las radiaciones ionizantes y ciertos fármacos y toxinas. En otros casos es la propia célula la que pone en marcha la apoptosis a través de las proteínas de la familia Bcl-2. Algunas de estas proteínas (p. e la Bax) alteran la integridad estructural y funcional

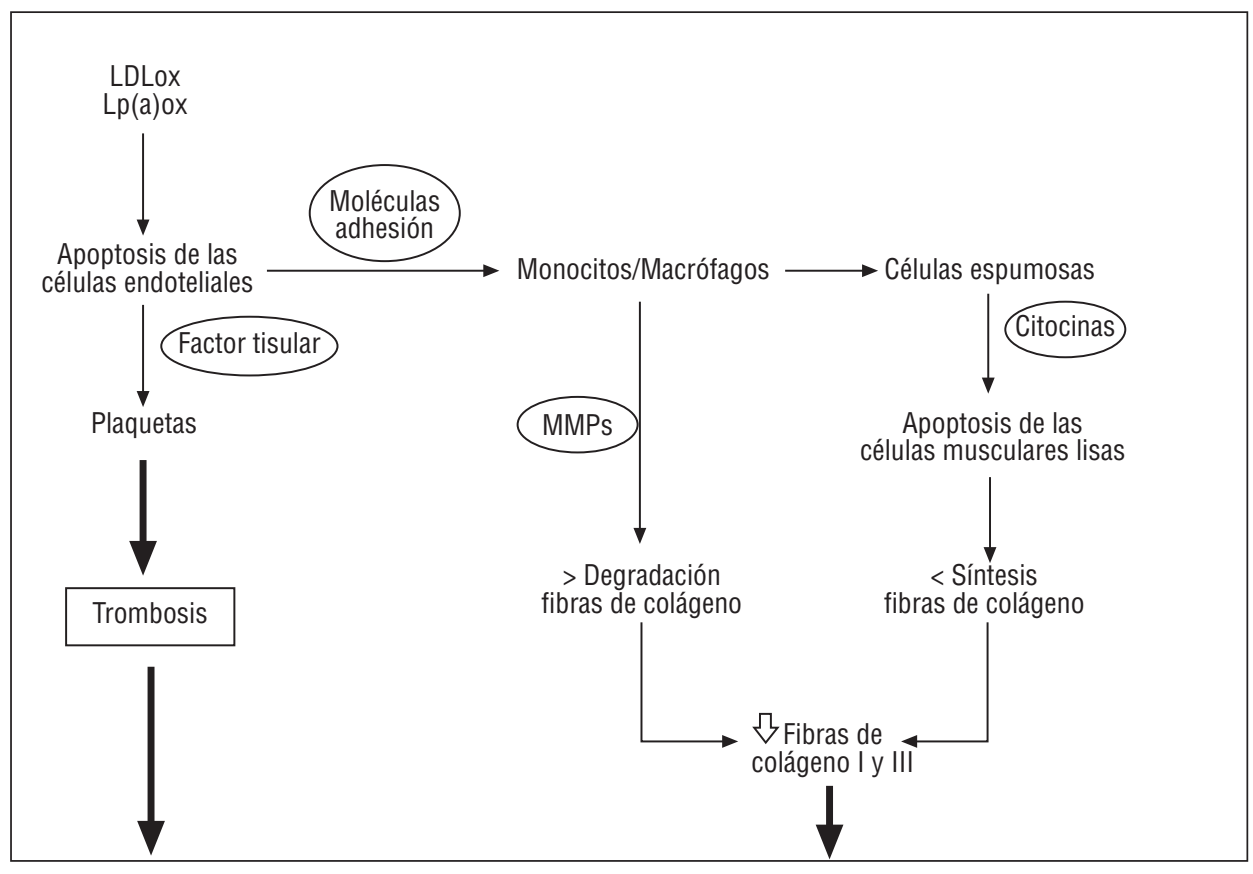

Fig. 1. Mecanismos moleculares que participan en la muerte de la célula por apoptosis. En trazo grueso se representa la membrana celular. $\mathrm{R}_{\mathrm{x}}$ : receptor específico para distintas factores extracelulares; TNFR: receptor del TNF. 
de la mitocondria, facilitando la liberación del citocromo c desde la cadena respiratoria hasta el citosol, donde activará a la proteasa APAF-1. Por el contrario, otras proteínas de la familia (p. ej., la Bcl-2) preservan la integridad mitocondrial. Así pues, del equilibrio entre las proteínas proapoptóticas y antiapoptóticas de la familia Bcl-2 resultará la activación o no de esta vía apoptótica.

Las proteínas del dominio de muerte y la APAF-1 activan a unas proteasas, caspasas, de las que las dos más importantes son la 3 o CPP32 y la 1 o enzima convertidora de la interleucina 1. Las caspasas activadas y otros factores proapoptóticos, como la ceramida y las especies reactivas del oxígeno, ponen en marcha una vía de señales que, a través de la elevación de la concentración de $\mathrm{Ca}^{2+}$ del citosol, conduce a la activación de enzimas citosólicas dependientes de $\mathrm{Ca}^{2+}$. Por otra parte, el daño de la cromatina nuclear provoca la expresión de ciertos protooncogenes que acaban induciendo la expresión de estas enzimas dependientes de $\mathrm{Ca}^{2+}$. Dichas enzimas ejecutan la destrucción de la célula y son de tres tipos: las endonucleasas que fragmentan el ADN, las proteasas que dañan el citoesqueleto y la transglutaminasa que provoca el entrecruzamiento de las proteínas del citoplasma y el arrugamiento de la célula.

Todos estos eventos intracelulares se acompañan de cambios en la composición de la membrana celular, incluyendo el aumento en la expresión de fosfatidil serina en la superficie externa de la misma. Dichos cambios posibilitan que los cuerpos apoptóticos que resultan de la fragmentación de la célula sean reconocidos, rápidamente captados e internalizados por los macrófagos adyacentes, impidiendo así la respuesta inflamatoria. La resolución de la apoptosis no comporta, pues, inflamación, a diferencia de lo que sucede en la oncosis.

\section{APOPTOSIS EN LAS ENFERMEDADES CARDIOVASCULARES}

El interés que actualmente despierta el estudio de la apoptosis en medicina es grande. En efecto, la apoptosis es un proceso fisiológico que durante el período prenatal y tras el nacimiento contrarresta los efectos de la proliferación celular, contribuyendo al mantenimiento de la masa celular y la arquitectura de los órganos y los tejidos. Por lo tanto, la disregulación de la apoptosis puede contribuir al desarrollo de diversas enfermedades ${ }^{8-10}$. Por un lado, la apoptosis exagerada puede conducir a la atrofia y a la insuficiencia funcional de un determinado órgano. Por otro lado, una apoptosis insuficiente de determinadas células puede participar en el desarrollo de ciertos procesos tumorales e inmunoinflamatorios.

En los últimos diez años se han publicado hallazgos que sugieren que la regulación de la apoptosis puede estar alterada en diversas enfermedades del corazón y de los vasos sanguíneos ${ }^{11}$ (tabla 2). A continuación se
TABLA 2. Enfermedades del corazón y de los vasos en las que se han descrito alteraciones de la apoptosis de las células cardiovasculares

\begin{tabular}{l}
\hline Enfermedades cardíacas \\
Cardiopatía isquémica \\
Cardiopatía hipertensiva \\
Miocardiopatías hereditarias \\
Miocardiopatía dilatada idiopática \\
Displasia arritmogénica del ventrículo derecho \\
Miocardiopatía hipertrófica familiar \\
Cardiopatías inmunológicas \\
Miocarditis \\
Rechazo del injerto cardíaco \\
Cardiopatías congénitas \\
Con alteraciones eléctricas \\
Con alteraciones vasculares \\
Cardiopatías tóxicas \\
Antraciclinas \\
Otras cardiopatías \\
Acromegalia \\
Enfermedades vasculares \\
Aterosclerosis \\
Arteriopatía hipertensiva \\
Reestenosis postangioplastia \\
Vasculopatías inmunológicas \\
Vasculitis \\
Vasculopatía del injerto \\
Otras vasculopatías \\
Aneurismas \\
Angiopatía diabética \\
\hline
\end{tabular}

consideran brevemente las posibles implicaciones fisiopatológicas del exceso o del defecto de la apoptosis en las células cardíacas y vasculares en algunas enfermedades del corazón y de los vasos.

\section{Apoptosis de los cardiomiocitos}

Durante el desarrollo prenatal del corazón, la desaparición de células por apoptosis desempeña un papel muy importante en su configuración morfológica definitiva $^{12}$. Además, se ha comprobado que el índice apoptótico de los corazones neonatos es mucho mayor en el ventrículo derecho que en el izquierdo, habiéndose propuesto que esta diferencia es la responsable de la diferenciación de la masa contráctil de ambos ventrículos, que es muy similar en el momento del nacimiento. Por otra parte, la degeneración reabsortiva del sistema de excitación y de conducción que sufren los corazones neonatos es el resultado de procesos apoptóticos. A partir de todo ello se entiende que algunas cardiopatías congénitas sean, en parte, el resultado de una apoptosis inadecuada durante este período de la vida ${ }^{12}$.

En el corazón adulto, el papel físiológico de la apoptosis de los cardiomiocitos está todavía sin aclarar. En la actualidad se dispone de datos que demuestran que el cardiomiocito es capaz de replicarse, al 


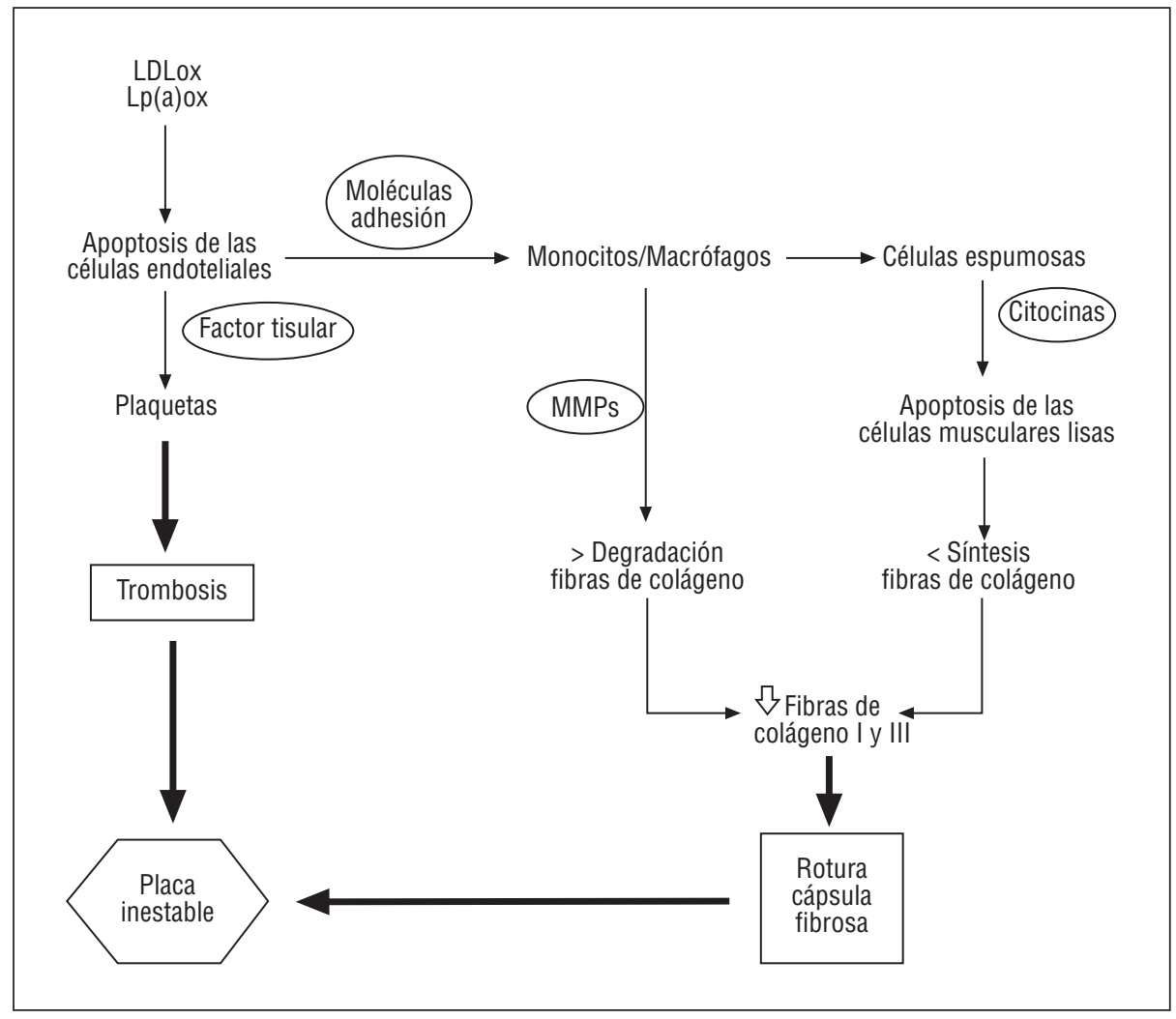

Fig. 2. Esquema representativo de la posible participación de la apoptosis de las células vasculares en la inestabilidad de las placas ateroscleróticas. LDLox: lipoproteínas de baja densidad oxidadas; Lp(a)ox: lipoproteína a oxidada; MMPs: metaloproteinasas de la matriz extracelular. igual que es capaz de entrar en apoptosis ${ }^{13}$, lo que sugiere que, como ocurre en la mayoría de los tejidos, la apoptosis y la proliferación de los cardiomiocitos son dos procesos que se complementan para regular la celularidad miocárdica. Por otra parte, se ha propuesto que la pérdida de cardiomiocitos debido a una excesiva apoptosis de los mismos podría ser un mecanismo determinante de la evolución de la disfunción ventricular a la insuficiencia cardíaca terminal en cardiopatías como la isquémica y la hipertensiva ${ }^{14}$.

En efecto, son muy abundantes los trabajos experimentales que describen la aparición de apoptosis en cardiomiocitos sometidos a isquemia y/o reperfusión ${ }^{15}$. Además, una exagerada apoptosis de los cardiomiocitos se ha objetivado en pacientes con infarto agudo de miocardio ${ }^{16,17}$ y con cardiopatía isquémica e insuficiencia cardíaca $^{18,19}$. Por otra parte, diversos estudios demuestran que la sobrecarga mecánica ${ }^{20}$ y los agonistas vasoconstrictores como la angiotensina $\mathrm{II}^{21}$ inducen la apoptosis de los cardiomiocitos. De hecho, la apoptosis de los cardiomiocitos es mayor en el ventrículo izquierdo de las ratas espontáneamente hipertensas (SHR) que en el de las ratas normotensas Wistar-Kyoto, especialmente cuando las primeras desarrollan insuficiencia cardíaca ${ }^{22}$. Queda por demostrar si la apoptosis puede ser la causa del menor número de cardiomiocitos presente en el ventrículo izquierdo de pacientes hipertensos sin enfermedad coronaria ${ }^{23}$ y si ello sería el resultado de una susceptibilidad exagerada de dichas células para morir vía apoptosis ${ }^{24}$.

\section{Apoptosis de las células musculares lisas vasculares}

La regulación de la proliferación y de la muerte por apoptosis en las células musculares lisas vasculares es un determinante importante en la configuración de la estructura normal de la pared vascular en condiciones fisiológicas. Por ello, el predominio de la proliferación sobre la apoptosis se ha propuesto como el mecanismo responsable de la acumulación de células musculares, que facilita el engrosamiento de la capa media y de la pared de las arterias pequeñas en la hipertensión arterial ${ }^{25}$ y que contribuye a la reestenosis tras la angioplastia ${ }^{26}$.

Por el contrario, se ha descrito que las células musculares lisas vasculares que han emigrado al espacio subintimal en una placa aterosclerótica presentan apoptosis más frecuentemente que las células que aún permanecen en la media ${ }^{27}$. La exagerada apoptosis de estas células puede ser un factor determinante de la vulnerabilidad de una placa aterosclerótica, pues comportaría una disminución de su número y una menor capacidad para la síntesis de colágeno fibrilar ${ }^{28}$. Ello, combinado con la liberación desde los macrófagos de las metaloproteinasas de matriz que degradan las fibras de colágeno, daría lugar a una cápsula fibrosa más frágil ${ }^{29}$ (fig. 2). En el desencadenamiento de la 
apoptosis de las células musculares se han implicado diversas citocinas secretadas por las células espumosas presentes en la lesión ${ }^{30,31}$.

\section{Apoptosis de las células endoteliales}

La apoptosis parece ser el principal mecanismo de muerte del endotelio en condiciones fisiológicas. Por otra parte, el equilibrio proliferación-apoptosis de las células endoteliales desempeña un papel crítico en la formación y regresión de los vasos sanguíneos, especialmente las arteriolas y los capilares. Por ello, una excesiva apoptosis de estas células puede estar implicada tanto en la disfunción endotelial, como en la inhibición de la angiogénesis, descritas en diversas enfermedades vasculares ${ }^{32}$.

Especialmente relevante puede ser la participación de la apoptosis endotelial en la patogenia de la aterosclerosis. Las células endoteliales apoptóticas presentan modificaciones fenotípicas de su superficie que se traducen en un aumento en la expresión de moléculas de adhesión $^{33}$ y de factor tisular procoagulante ${ }^{34}$. Lo primero participaría en los estadios iniciales de la formación de la lesión aterosclerótica, mientras que lo segundo contribuiría a la complicación trombótica de la misma (fig. 2). Las lipoproteínas aterogénicas, especialmente las formas oxidadas, podrían ser las inductoras de la apoptosis de las células endoteliales en el contexto de la aterosclerosis ${ }^{35-37}$.

\section{DESARROLLOS FUTUROS}

Los primeros trabajos sobre apoptosis y enfermedades cardiovasculares se han publicado durante los últimos 5 años. De ello se deduce que éste es un campo en el que todo está prácticamente por desarrollarse. En este sentido, se pueden avanzar ciertas consideraciones con posible interés fisiopatológico, diagnóstico y terapéutico.

\section{En el ámbito de los mecanismos de la apoptosis}

Desde el punto de vista fisiológico, la apoptosis es un proceso defensivo de eliminación de células viejas, dañadas o infectadas. Así, se ha descrito que la liberación experimental de virus en el miocardio se acompaña de la apoptosis de los cardiomiocitos infectados ${ }^{38}$. En los cardiomiocitos de las ratas SHR se ha demostrado la presencia de material genético retroviral, sin relación aparente con la sobrecarga hemodinámica ventricular ${ }^{39}$. También se ha detectado genoma adenoviral en el miocardio de pacientes con miocardiopatía dilatada idiopática que no presentaban signos de miocarditis activa ${ }^{40}$. Sería, pues, interesante delimitar la participación de la infección viral en la exagerada

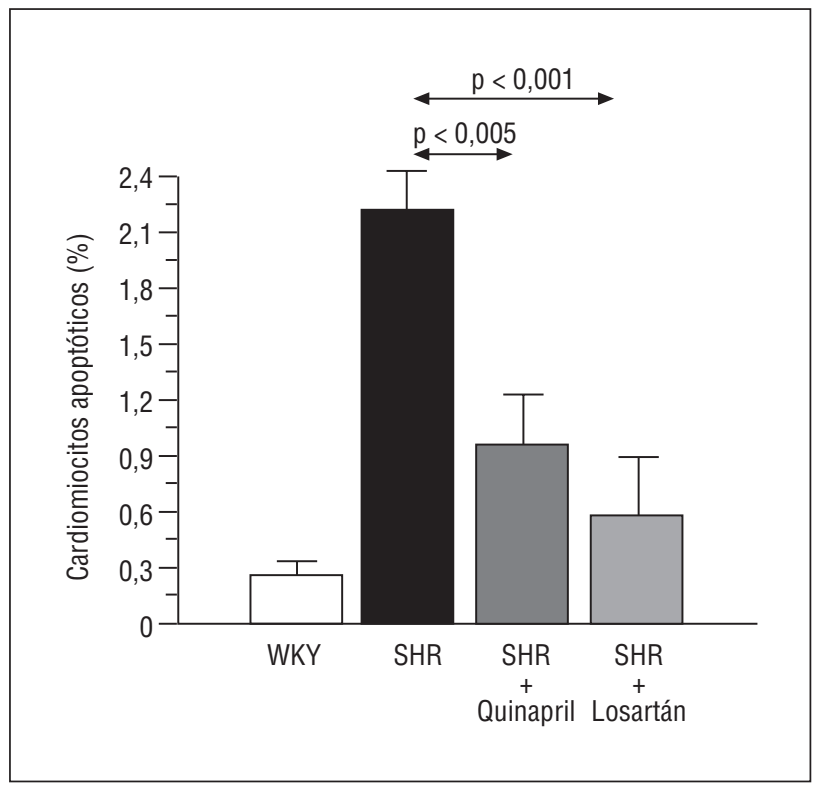

Fig. 3. Efectos in vivo del inhibidor de la ECA quinapril y del antagonista de los receptores $A T_{1}$ losartán sobre la apoptosis de los cardiomiocitos en la rata espontáneamente hipertensa. WKY: ratas normotensas Wistar-Kyoto; SHR: ratas espontáneamente hipertensas. Adaptada de referencias 50 y 51.

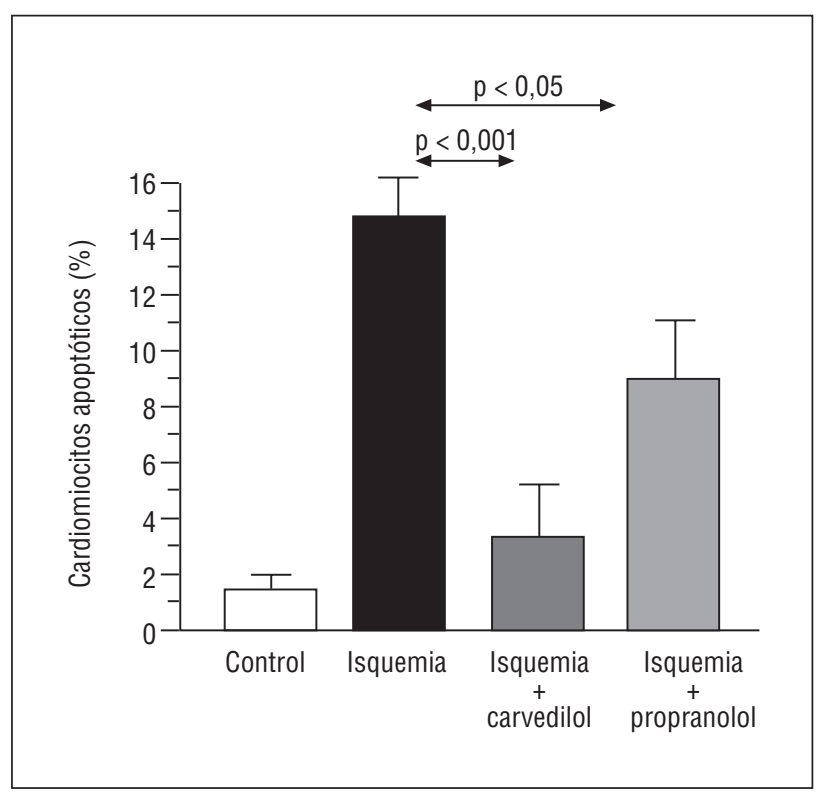

Fig. 4. Efectos in vitro de los $\beta$ bloqueantes carvedilol y propranolol sobre la apoptosis de los cardiomiocitos en el conejo sometido a isquemia/reperfusión miocárdica. Adaptada de referencia 52.

apoptosis de los cardiomiocitos descrita tanto en las ratas $\mathrm{SHR}^{22}$ como en los pacientes con miocardiopatía idiopática dilatada ${ }^{18,19}$.

Diversas evidencias sugieren que la estimulación de los receptores $\beta$ adrenérgicos da lugar a la apoptosis de los cardiomiocitos ${ }^{41}$. Recientemente, se ha demostrado que este efecto apoptótico de las catecolaminas 
está mediado por la subunidad Gs $\alpha$ de las proteínas $\mathrm{G}$ acopladas al receptor $\beta$ adrenérgico ${ }^{42}$. Existe controversia sobre si la estimulación crónica de los receptores $\beta$ adrenérgicos o lo opuesto, su desensibilización, es beneficioso o perjudicial en la patogenia de la insuficiencia cardíaca. La posibilidad de que la estimulación $\beta$ adrenérgica facilite la pérdida de cardiomiocitos vía apoptosis sugeriría que es perjudicial.

Es bien sabido que la angiotensina II estimula el crecimiento de las células cardíacas y de las células vasculares. Pero datos recientes demuestran que también puede facilitar la apoptosis de las mismas. Mientras que la apoptosis de las células endoteliales y de las células musculares lisas vasculares estaría mediada por los receptores $\mathrm{AT}_{2}^{43}$, la de los cardiomiocitos resultaría de la interacción del péptido con los receptores $\mathrm{AT}_{1}{ }^{21,44}$. La comprensión del equilibrio entre las acciones procrecimiento y proapoptosis de la angiotensina II en el sistema cardiovascular es importante para entender su contribución al remodelado cardiovascular que se da en distintas enfermedades.

Fisiológicamente, el óxido nítrico es un regulador bifuncional de la apoptosis en las células endoteliales ${ }^{45}$. En condiciones fisiológicas ese efecto antiapoptótico se ejerce a través de varios mecanismos, fundamentalmente inhibiendo a las caspasas mediante su S-nitrosilación ${ }^{46}$. Sin embargo, en condiciones patológicas caracterizadas por producción exagerada de anión superóxido, el óxido nítrico puede ser la fuente para la formación de peroxinitritos, que inducen el proceso de la apoptosis ${ }^{47}$. Por lo tanto, desde el punto de vista de la apoptosis, aún está por dilucidar el posible papel citotóxico del óxido nítrico en enfermedades como la aterosclerosis y la hipertensión, caracterizadas por la formación excesiva de especies reactivas del oxígeno en la pared vascular.

\section{En el ámbito de la detección de la apoptosis}

La detección in situ de las células apoptóticas presenta actualmente varias limitaciones, derivadas de la propia biofisiología del proceso: su corta duración, que afecta a células aisladas y que tras su resolución por fagocitosis no persisten lesiones residuales. Por lo tanto, la identificación y la cuantificación de la apoptosis en los tejidos requiere estudios muy minuciosos y exhaustivos. A ello se añade que ninguna de las técnicas bioquímicas e histológicas hoy día utilizadas es suficientemente específica o sensible, por lo que se recomienda el uso combinado de varias de ellas ${ }^{14,48}$ : la electroforesis en gel para detectar la presencia de la fragmentación apoptótica del ADN que da lugar a la imagen típica en escalera, las técnicas inmunohistoquímicas destinadas a marcar en las células los extremos 3' de los fragmentos del ADN (Taq polimerasa, transferasa deoxinucleótido terminal, $P f u$ polimerasa) o a identificar en la membrana celular sustancias como la annexina y las técnicas que evalúan la expresión y la actividad de las caspasas.

Para el diagnóstico sistémico de la apoptosis se pueden detectar en sangre ciertos marcadores del proceso apoptótico. Así, en pacientes con hipertensión arterial se han descrito alteraciones de las concentraciones sanguíneas de la proteína antiapoptótica Bcl-2 ${ }^{49}$, mientras que en pacientes con insuficiencia cardíaca se han descrito alteraciones de los niveles circulantes de las proteínas del sistema $\mathrm{Fas}^{50}$. Aunque se trata de datos muy preliminares, una nueva vía se está abriendo para poder evaluar el estado proapoptótico o antiapoptótico de los pacientes. Está claro que en este campo el desarrollo ha de venir dado por el desarrollo de marcadores específicos del proceso apoptótico en el sistema cardiovascular.

\section{En el ámbito de la modulación farmacológica de la apoptosis}

En distintos modelos experimentales in vivo e in vitro se ha observado que algunos de los fármacos que se utilizan actualmente para el tratamiento de las enfermedades cardiovasculares modifican la apoptosis. Los inhibidores de la $\mathrm{ECA}^{51}$ y los antagonistas de los receptores $\mathrm{AT}_{1}$ de la angiotensina ${ }^{52}$ previenen la apoptosis de los cardiomiocitos en la cardiopatía hipertensiva (fig. 3). Los $\beta$ bloqueantes protegen de la apoptosis a los cardiomiocitos en condiciones de isquemia-reperfusión ${ }^{53}$ (fig. 4). Los antagonistas del calcio podrían prevenir la evolución de las lesiones ateroscleróticas impidiendo la apoptosis de las células vasculares $^{54}$. El papel de los inhibidores de la hidroximetil-glutaril coenzima A es más controvertido, pues mientras que los compuestos lipofílicos (atorvastatina, simvastatina, lovastatina) estimulan la apoptosis de las células musculares lisas vasculares, los compuestos hidrofílicos (pravastatina) no la modifican ${ }^{55}$.

La modulación de la apoptosis basada en el diseño de nuevas estrategias farmacológicas empieza a emerger como un área terapéutica prometedora. En experimentos efectuados en ratas sometidas a isquemia-reperfusión miocárdica se ha observado que la transfección previa del gen antiapoptótico $b c l-2$ cursaba con menor apoptosis de los cardiomiocitos, menor liberación de enzimas cardiomiocitarias a la sangre y menor compromiso de la función cardíaca que en las ratas no transfectadas $^{56}$. En un modelo similar de daño isquémico miocárdico se ha demostrado que un inhibidor de las caspasas, el ZVAD-fmk, disminuye la apoptosis de los cardiomiocitos y reduce el área de infarto con respecto a los animales no tratados ${ }^{57}$. En roedores tratados con el factor de crecimiento similar a la insulina tipo 1, por vía sistémica ${ }^{58}$ o mediante transfección ${ }^{59}$, se constató una menor aparición de apoptosis miocárdica tras la inducción del infarto que en los animales no tratados. Además, los animales que recibieron el factor presenta- 
ron menos remodelado de la pared ventricular y menos compromiso de la función ventricular que los animales sin tratar. Aunque todavía no está suficientemente aclarado el mecanismo del efecto antiapoptótico del factor de crecimiento, algunos datos sugieren que podría estar relacionado con la inducción de alguna proteína antiapoptótica de la familia $\mathrm{Bcl}-2$ y/o con la inhibición de alguna de las caspasas efectoras de la destrucción celular ${ }^{60}$.

\section{CONCLUSIONES}

Uno de los retos principales de la medicina cardiovascular para las próximas décadas es el de prevenir el desarrollo de la insuficiencia cerebral, la insuficiencia cardíaca, la insuficiencia renal y la insuficiencia circulatoria periférica que pueden desarrollar los pacientes afectos de enfermedades cardiovasculares.

En el caso de la insuficiencia cardíaca, existen abundantes indicios de que su magnitud epidemiológica le va a conferir el carácter de una verdadera epidemia en los años venideros. Para minimizar el impacto de tal epidemia será preciso incrementar nuestros conocimientos sobre los mecanismos que intervienen en el inicio y la evolución del deterioro funcional del miocardio. En este sentido, en su último informe, el National Heart, Lung, and Blood Institute Special Emphasis Panel on Heart Failure Research proponía como una de las líneas de investigación prioritarias de la insuficiencia cardíaca el estudio de la contribución de las alteraciones de la apoptosis a la progresiva pérdida de músculo cardíaco y a la modificación estructural y funcional (remodelado) del músculo residual ${ }^{61}$.

Sin embargo, justo es reconocer que el campo de la apoptosis del sistema cardiovascular presenta todavía serias limitaciones. Algunas hacen referencia a lo fragmentario de los datos disponibles. Otras a la dificultad de valorar con precisión la ocurrencia del proceso apoptótico en los tejidos. Finalmente, las hay que se refieren a nuestro escaso conocimiento de los mecanismos que realmente gobiernan la apoptosis en condiciones normales y patológicas. La superación de esas dificultades puede hacer de la apoptosis un ejemplo paradigmático del enfoque celular-molecular que hoy en día se impone para la comprensión de las enfermedades del corazón y de los vasos sanguíneos.

\section{BIBLIOGRAFÍA}

1. Kerr JFR, Wyllie AH, Currie AR. Apoptosis: a basic biological phenomenon with wide-ranging implication in tissue kinetics. $\mathrm{Br}$ J Cancer 1972; 26: 239-257.

2. Majno G, Joris I. Apoptosis, oncosis, and necrosis. Am J Pathol 1995; 146: 3-15.
3. Didenko W, Hornsby PJ. Presence of double-strand breaks with single-base 3' overhangs in cells undergoing apoptosis but not necrosis. J Cell Biol 1996; 135: 1369-1376.

4. Eguchi Y, Shimizu S, Tsujimoto Y. Intracellular ATP levels determine cell death fated by apoptosis or necrosis. Cancer Res 1997; 57: 1835-1840.

5. Granville DJ, Carthy CM, Hunt DWC, McManus BM. Apoptosis: molecular aspects of cell death and disease. Lab Invest 1998; 78: 893-913.

6. Saini KS, Walker NI. Biochemical and molecular mechanisms regulating apoptosis. Mol Cell Biochem 1998; 178: 9-25.

7. Vaux DL, Strasser A. The molecular biology of apoptosis. Proc Natl Acad Sci USA 1996; 93: 2239-2244.

8. Hetts SW. To die or not to die: an overview of apoptosis and its role in disease. JAMA 1998; 279: 300-307.

9. Thatte U, Dahanukar S. Apoptosis: clinical relevance and pharmacological manipulation. Drugs 1997; 54: 511-532.

10. Thompson CB. Apoptosis in the pathogenesis and treatment of disease. Science 1995; 267: 1456-1461.

11. Díez J, Fortuño MA, González A, Ravassa S. Apoptosis y Enfermedades Cardiovasculares. Madrid: Ergón, 1999.

12. James TN. Normal and abnormal consequences of apoptosis in the human heart. From postnatal morphogenesis to paroxysmal arrhythmias. Circulation 1994; 90: 556-573.

13. Anversa P, Kajstura J. Ventricular myocytes are not terminally differentiated in the adult mammalian heart. Circ Res 1998; 83: 1-14.

14. Anversa P, Leri A, Beltrami CA, Guerra S, Kajstura J. Myocyte death and growth in the failing heart. Lab Invest 1998; 78: 767786.

15. Buja LM, Entman ML. Modes of myocardial cell injury and cell death in ischemic heart disease. Circulation 1998; 98: 1355-1357.

16. Itoh G, Tamura J, Suzuki M, Suzuki Y, Ikeda H, Koibe M et al. DNA fragmentation of human infarcted myocardial cells demonstrated by the nick end labeling method and DNA agarose gel electrophoresis. Am J Pathol 1995; 146: 1325-1331.

17. Saraste A, Pulkki K, Kallajoki M, Henriksen K, Parvinen M, Voipio-Pulkki LM. Apoptosis in human acute myocardial infarction. Circulation 1997; 95: 320-333.

18. Narula J, Haider N, Virmani R, Disalvo G, Kolodgie FD, Hajjar $\mathrm{RJ}$ et al. Apoptosis in myocytes in end-stage heart failure. $\mathrm{N}$ Engl J Med 1996; 335: 1182-1189.

19. Olivetti G, Abbi R, Quaini F, Kajstura J, Cheng W, Nitahara JA et al. Apoptosis in the failing human heart. N Engl J Med 1997; 336: 1131-1141.

20. Cheng W, Li B, Kajstura J, Li P, Wolin MS, Sonnenblick EH et al. Strech-induced programmed myocyte cell death. J Clin Invest 1995; 96: 2247-2259.

21. Kajstura J, Cigola E, Malhotra A, Li P, Cheng W, Meggs LG et al. Angiotensin II induces apoptosis of adult ventricular myocytes in vitro. J Mol Cell Cardiol 1997; 29: 859-870.

22. Díez J, Fortuño MA, Ravassa S. Apoptosis in hypertensive heart disease. Curr Opin Cardiol 1998; 13: 317-325.

23. Olivetti G, Melissari M, Balbi T, Quaini F, Cigola E, Sonnenblick EH et al. Myocyte cellular hypertrophy is responsible for ventricular remodelling in the hypertrophied heart of middle aged individuals in the absence of cardiac failure. Cardiovasc Res 1994; 28: 1199-1208.

24. Díez J, Fortuño MA, Ravassa S. Apoptosis en la cardiopatía hipertensiva. Rev Esp Cardiol 1999; 52 (Supl 3): 19-25.

25. Hamet P. Proliferation and apoptosis of vascular smooth muscle in hypertension. Curr Opin Nephrol Hypertens 1995; 4: 1-7.

26. Isner JM, Kearney M, Bortman S, Passeri J. Apoptosis in human atherosclerosis and restenosis. Circulation 1995; 91: 2703-2711.

27. Best PJM, Hasdai D, Sangiorgi G, Schwartz RS, Holmes DR Jr, Simari RD et al. Apoptosis. Basic concepts and implications in coronary artery disease. Arterioscler Thromb Vasc Biol 1999; 19: 14-22.

28. Kockx MM. Apoptosis in the atherosclerotic plaque. Quantitative and qualitative aspects. Arterioscler Thromb Vasc Biol 1998; 18: 1519-1522. 
29. Kockx MM, Herman AG. Apoptosis in atherogenesis: implications for plaque destabilization. Eur Heart J 1998; 19 (Supl G): G23-G28.

30. Geng YG, Libby P. Evidence for apoptosis in advanced human atheroma: colocalization with interleukin $1 \beta$ converting enzyme. Am J Pathol 1995; 147: 251-266.

31. Geng YG, Wu Q, Muszynski M, Hansson GK, Libby P. Apoptosis of vascular smooth muscle cells induced by in vitro stimulation with IFN- $\gamma$, TNF- $\alpha$, and IL-1 $\beta$. Arterioscler Thromb Vasc Biol 1996; 16: 19-27.

32. Karsan A, Harlan JM. Modulation of endothelial cell apoptosis: mechanisms and pathophysiological roles. J Atheroscler Thromb 1996; 3: 75-80.

33. Hebert MJ, Gullans SR, Mackenzie HS, Brady HR. Apoptosis of endothelial cells is associated with paracrine induction of adhesion molecules: evidence for an interleukin-1beta-dependent paracrine loop. Am J Pathol 1998; 152: 523-532.

34. Bombeli T, Karsan A, Tait JF, Harlan JM. Apoptotic vascular endothelial cells become procoagulant. Blood 1997; 89: 2429-2442.

35. Dimmeler S, Haendeler J, Galle J, Zeiher AM. Oxidized low-density lipoprotein induces apoptosis of human endothelial cells by activation of CPP32-like proteases. A mechanistic clue to the «response to injury» hypothesis. Circulation 1997; 95: 17601763.

36. Escargueil-Blanc I, Meilhac O, Pieraggi MT, Arnal JF, Salvayre R, Negre-Salvayre A. Oxidized LDLs induce massive apoptosis of cultured human endothelial cells through a calcium-dependent pathway. Arterioscler Thromb Vasc Biol 1997; 17: 331-339.

37. Galle J, Schneider R, Heinloth A, Wanner C, Galle PR, Conzelman $\mathrm{E}$ et al. $\mathrm{Lp}(\mathrm{a})$ and LDL induce apoptosis in human endothelial cells and in rabbit aorta: Role of oxidative stress. Kidney Int 1999; 55: 1450-1461.

38. Agah R, Kirshenbaum LA, Abdellatif M, Truong LD, Chakraborty S, Michael LH et al. Adenoviral delivery of E2F-1 directs cell cycle reentry and p53-independent apoptosis in postmitotic adult myocardium in vivo. J Clin Invest 1997; 100: 2722-2728.

39. Sirokman GF, Humphries DE, Bing OHL. Endogenous retroviral transcripts in myocytes from spontaneously hypertensive rats. Hypertension 1997; 30: 88-93.

40. Pauschinger M, Bowles EN, Fuentes-García J, Pham V, Kühl U, Schwimmbeck PL et al. Detection of adenoviral genome in the myocardium of adult patients with idiopathic left ventricular dysfunction. Circulation 1999; 99: 1348-1354.

41. Communal C, Singh K, Pimentel DR, Colucci WS. Norepinephrine stimulates apoptosis in adult rat ventricular myocytes by activation of the beta-adrenergic pathway. Circulation 1998; 98: 1329-1334.

42. Geng YJ, Ishikawa Y, Vatner DE, Wagner TE, Bishop SP, Vatner SF et al. Apoptosis of cardiac myocytes in Gs $\alpha$ transgenic mice. Circ Res 1999; 84: 34-42.

43. Horiuchi M, Akishita M, Dzau VJ. Recent progress in angiotensin II type 2 receptor research in the cardiovascular system. Hypertension 1999; 33: 613-621.

44. Cigola E, Kajstura J, Li B, Meggs LG, Anversa P. Angiotensin II activates programmed cell death in vitro. Exp Cell Res 1997;
231: 363-371.

45. Kim YM, Bombeck CA, Billiar TR. Nitric oxide as a bifunctional regulator of apoptosis. Circ Res 1999; 84: 253-256.

46. Tzeng E, Kim YM, Pitt BR, Lizonova A, Kovesdi I, Billiar TR. Adenoviral transfer of the inducible nitric oxide synthase gene blocks endothelial cell apoptosis. Surgery 1997; 122: 255-263.

47. Messmer UK, Ankarcrona M, Nicotera P, Brune B. p53 expression in nitric-oxide induced apoptosis. FEBS Lett 1994; 355: 23 26.

48. Buja LM. Modulation of the myocardial response to ischemia. Lab Invest 1998; 78: 1345-1373.

49. Buemi M, Allegra A, Corica F, Aloisi C, Ruello A, Giacobbe MS et al. Recluced bcl-2 concentrations in hypertensive patients after lisinopril or nifedipine administration. Am J Hypertens 1999; 12: 73-75.

50. Nishigaki K, Minatoguchi S, Seishima M, Asano K, Noda T, Yasuda $\mathrm{N}$ et al. Plasma Fas ligand, an inducer of apoptosis, and plasma soluble Fas, an inhibitor of apoptosis, in patients with chronic congestive heart failure. J Am Coll Cardiol 1997; 29: 1214-1220.

51. Díez J, Panizo A, Hernández M, Vega F, Sola I, Fortuño MA et al. Cardiomyocyte apoptosis and cardiac angiotensin-converting enzyme in spontaneously hypertensive rats. Hypertension 1997; 30: 1029-1034.

52. Fortuño MA, Ravassa S, Etayo JC, Díez J. Overexpression of Bax protein and enhanced apoptosis in the left ventricle of spontaneously hypertensive rats. Effects of $\mathrm{AT}_{1}$ blockade with losartan. Hypertension 1998; 32: 280-286.

53. Yue T-L, Ma X-L, Wang X, Romanic AM, Liu G-L, Louden C et al. Possible involvement of stress-activated protein kinase signaling pathway and Fas receptor expression in prevention of ischemia/reperfusion-induced cardiomyocyte apoptosis by carvedilol. Circ Res 1998; 82: 166-174.

54. Díez J. Calcium channel antagonists. The future. En: Heagerty A, editor. Review of Calcium Channel Antagonists. Wiltshire: Euromed Communications Ltd., 1998; 65-76.

55. Guijarro C, Blanco-Colio LM, Ortego M, Alonso C, Ortiz A, Plaza JJ et al. 3-Hydroxy-3-methyl-glutaryl coenzyme a reductase and isoprenylation inhibitors induce apoptosis of vascular smooth muscle cells in culture. Circ Res 1998; 83: 490-500.

56. Sawa Y, Bai HZ, Suzuki K, Tsujimoto Y, Matsuda H. Overexpression of bcl-2 gene improves the myocardial tolerance to ischemia-reperfusion by preventing DNA fragmentation. Circulation 1995; 92: I-772.

57. Yaoita H, Ogawa K, Maehara K, Maruyama Y. Attenuation of ischemia/reperfusion injury in rats by a caspase inhibitor. Circulation 1998; 97: 276-281.

58. Buerke M, Murohara T, Skurk C, Nuss C, Tomaselli K, Lefer AM. Cardioprotective effect of insulin-like growth factor I in myocardial ischemia followed by reperfusion. Proc Natl Acad Sci USA 1995; 92: 8031-8035.

59. Li Q, Li B, Wang X, Leri A, Jana KI, Liu Y et al. Overexpression of insulin-like growth factor-1 in mice protects from myocyte death after infarction, attenuating ventricular dilation, wall stress, and cardiac hypertrophy. J Clin Invest 1997; 100: 1991-1999.

60. Parrizas M, Le Roith D. Insulin-like growth factor-1 inhibition of apoptosis is associated with increased expression of the bcl-xL gene product. Endocrinology 1997; 138: 1355-1358.

61. Cohn JN, Bristow MR, Chien KR, Colucci WS, Frazier OH, Leinwand LA et al. Report of the National Heart, Lung, and Blood Institute Special Emphasis Panel on Heart Failure Research. Circulation 1997; 95: 766-770. 